\title{
Bispectral Analysis of Harmonic Oscillations Measured using Beam Emission Spectroscopy and Magnetic Probes in CHS
}

\author{
Tetsutarou OISHI ${ }^{a}$, Shinichiro KADO ${ }^{1), b}$, Mikirou YOSHINUMA, Katsumi IDA, \\ Tsuyoshi AKIYAMA, Takashi MINAMI, Kenichi NAGAOKA, Akihiro SHIMIZU, \\ Shoichi OKAMURA and CHS group \\ National Institute for Fusion Science, 322-6 Oroshi-cho, Toki 509-5292, Japan \\ ${ }^{1)}$ High Temperature Plasma Center, The University of Tokyo, 5-3-5 Kashiwanoha, Kashiwa 277-8568, Japan
}

(Received 16 November 2007 / Accepted 19 February 2008)

\begin{abstract}
The coherent MHD oscillation, which consists of the fundamental frequency of several kilohertz and its higher harmonics, (harmonic oscillation: HO) has been observed in Compact Helical System. HO consists of two pairs of harmonic series. One is located in the core region near the $\iota=0.5$ rational surface (denoted as "HO (core)"), the other is located in the edge region near the $\iota=1.0$ rational surface (denoted as "HO (edge)"). In the present study, bispectral analysis is applied to the fluctuation data, for which HO is measured by beam emission spectroscopy (BES) and using magnetic probes. The analysis has revealed that fundamental mode of HO in both the magnetic and core density fluctuations have phase correlation with the harmonics including fundamental oscillation, while HO in edge density fluctuation does not have such phase correlation. Mode numbers of HOs are identical for harmonic components having different frequencies, i.e., $m / n=-2 / 1$ for HO (core) and $m / n=-1 / 1$ for $\mathrm{HO}$ (edge). It suggests that the generation of harmonics cannot be interpreted simply as mode coupling because the summation rule for the wavenumber is not satisfied, even though the bicoherence value is significant. The bicoherence value and relative amplitude of higher harmonics correlate with each other, which suggests that bicoherence indicates the degree of distortion of the signals.
\end{abstract}

(C) 2008 The Japan Society of Plasma Science and Nuclear Fusion Research

Keywords: bispectral analysis, bicoherence, fluctuation, magnetohydrodynamics, edge harmonic oscillation, Compact Helical System, beam emission spectroscopy, edge transport barrier

DOI: $10.1585 /$ pfr.3.S1010

\section{Introduction}

Fluctuations of plasma parameters have been recognized to correlate with the properties of plasma confinement. Characteristics of the fluctuations include the amplitude and level, frequency and wavenumber spectrum, spatial structure represented by radial locality or poloidal/toroidal mode number $(m / n)$, and so on. In addition to these characteristics, recently, attention has been paid to the nonlinear characteristics such as the correlation between spectral components having different frequencies. In order to investigate the nonlinear characteristics of the fluctuations, bispectal analysis is considered to be useful [1]. Bispectral analysis has been applied to several tokamaks [2], helicals [3], and other types of plasma confinement devices to investigate the interaction among turbulence, magnetohydrodynamic (MHD) oscillations, and zonal flows [4-6].

Recently, a coherent MHD mode, which consists of a fundamental frequency of several kilohertz and its higher

\footnotetext{
author'se-mail: t-oishi@mail.nucl.nagoya-u.ac.jp

a) present address: Nagoya University, Chikusa-ku, Nagoya 464-8603, Japan

b) present address: The University of Tokyo, 7-3-1 Hongo, Bunkyo-ku, Tokyo 113-8656, Japan, kado@n.t.u-tokyo.ac.jp
}

harmonics, (harmonic oscillation: $\mathrm{HO}$ ) $[7,8]$ has been observed in edge transport barrier (ETB) discharges of Compact Helical System (CHS), which is a low-aspect-ratio, middle-sized heliotron (major radius $=1.0 \mathrm{~m}$, minor radius $=0.2 \mathrm{~m}$, toroidal period number $=8$, and polarity $=$ 2) [7,9-13]. The characteristics of this mode have been investigated from the viewpoint of a comparison with an edge harmonic oscillation (EHO) [14-17], which is recognized to have a role in enhancing particle transport in the quiescent H-mode of tokamaks. Fluctuation measurement by beam emission spectroscopy (BES) $[18,19]$ and using magnetic probe (MP) array [20] have revealed that HO in CHS consists of two pairs of harmonic series located in different radial positions. One is located in the core region of normalized minor radius $\rho=0.4-0.5$ near the $\iota=0.5$ rational surface (denoted as HO (core)), the other is located in the edge region of $\rho=0.9-1.0$ near $\iota=1.0$ rational surface (denoted as HO (edge)). All harmonic components of both $\mathrm{HO}$ (core) and $\mathrm{HO}$ (edge) have identical mode numbers, namely, $m / n=-2 / 1$ for $\mathrm{HO}$ (core) and $m / n=-1 / 1$ for HO (edge), where negative $m$ indicates the electron diamagnetic direction and positive $n$ indicates the toroidal field direction, even though the frequency of $p$ th harmonic is $p$ times larger than that of 1 st harmonic 
for both $\mathrm{HO}$ (core) and $\mathrm{HO}$ (edge), where $p$ is a positive integer [21].

In the present study, bispectral analysis is applied to the fluctuation data for which HO is measured by BES and using MPs in order to investigate the correlation between the harmonic components.

\section{Experimental Setup}

BES has been performed in CHS to simultaneously measure both local density fluctuations and gradients. BES detects emissions from the collisionally excited neutral beam atoms (denoted as "beam emission") [22]. The spatial channels used in BES consist of 16 optical fibers with an objective lens. In the present experiment, $\mathrm{H}_{\alpha}$ beam emission from the intersection of the neutral atomic hydrogen beam used for heating and the sightline for each fiber channel was measured. One can select the arrangement of the fibers in the radial or poloidal direction to measure each structure of the fluctuations.

Magnetic probe array was also used to measure both the poloidal and toroidal structures of the magnetic fluctuations. The poloidal magnetic probe array consists of sixteen probes covering a poloidal angle $\Delta \theta=235.5 \mathrm{deg}$. We define the absolute poloidal coordinate as $\theta=0 \mathrm{deg}$. and $180 \mathrm{deg}$. at the midplane outside and inside the torus, respectively [23]. Five toroidal magnetic probes covering the toroidal angle $\Delta \phi=\pi$ were located at every $\pi / 4$ section at the major radius $R=1.2 \mathrm{~m}$ and $\theta=320 \mathrm{deg}$.

\section{Data Analysis Method}

When a wave with a frequency $f$ is phase correlated with spectral components with frequencies $f_{1}$ and $f_{2}$ satisfying the summation rule $f=f_{1} \pm f_{2}$, we can use the bicoherence value as an indicator of phase correlation among these three waves [1]. Bicoherence $b^{2}\left(f_{1}, f_{2}\right)$ for the waves having the frequencies $f, f_{1}$, and $f_{2}$ is defined as

$$
b^{2}\left(f_{1}, f_{2}\right)=\frac{\left|<X\left(f_{1}\right) X\left(f_{2}\right) X *(f)>\right|^{2}}{<\left|X\left(f_{1}\right) X\left(f_{2}\right)\right|^{2}><|X(f)|^{2}>},
$$

where $X(f)$ is the Fourier transform of the time trace $x(t)$ and $<>$ indicates ensemble averaging. In the present study, the fast Fourier transform was performed every $2 \mathrm{~ms}$ having 2000 and 1000 data points for BES and MP measurements, respectively, with a frequency resolution $\Delta f$ of $0.5 \mathrm{kHz}$. The significant level of $b^{2}\left(f_{1}, f_{2}\right)$ is given by $1 / M$ where $M$ is the number of ensembles averaged to obtain the bicoherence spectrum [1]. In our case, $M$ is 15 so that the significant level of $b^{2}\left(f_{1}, f_{2}\right)$ is $1 / 15=0.067$.

Wave-wave coupling, i.e., the wave having the frequency $f$ is generated by the coupling between the waves having the frequencies $f_{1}$ and $f_{2}$, is the representative case for which the bicoherence value becomes significant. However, the wave-wave coupling is not necessarily the only mechanism responsible for the phase correlation revealed by bicoherence analysis. Summation rules concern- ing both frequency $f=f_{1} \pm f_{2}$ and wavenumber $k=k_{1} \pm k_{2}$ must be obeyed to generate the resulting wave by wavewave coupling.

\section{Experimental Results}

Figure 1 shows typical experimental conditions and temporal evolution of plasma parameters for a discharge showing harmonic oscillation: (a) heating condition, (b) $\mathrm{H}_{\alpha}$ intensity, (c) magnetic fluctuation, and (d) frequency spectrum of magnetic fluctuation. Plasma was generated by electron cyclotron heating and was further heated using two neutral beam injection (NBI) systems. Additional gas was introduced as a means to increase density. The standard magnetic field configuration of CHS was applied, in which the vacuum magnetic axis position $R_{\mathrm{ax}}$ was $92.1 \mathrm{~cm}$ from the torus center, the toroidal magnetic field strength at the magnetic axis $B_{\mathrm{ax}}$ was $0.95 \mathrm{~T}$, and the magnitude of the quadrupole field $B_{\mathrm{q}}$, denoting the degree of cancellation of the intrinsic quadrupole component formed by the helical coils, was $-50 \%$. In cases in which the heating power exceeds a certain threshold, ETB characterized by a sudden drop in the $\mathrm{H}_{\alpha}$ intensity signal, as shown in Fig. 1(b), is formed. It has been found that the line-averaged density, the edge density gradient, and the stored energy begin to increase at the ETB transition [7,9-13]. In the latter half of the phase having ETB, HO consisting of a 1st harmonic with the frequency of $4.0 \mathrm{kHz}$ and a 2 nd harmonic appears in the magnetic fluctuation, as shown in Figs. 1 (c) and (d). It is known that this oscillation is identical to HO (core) [21].

We applied bispectral analysis to the fluctuation data having harmonic oscillation measured by BES and using MPs. Figure 2 shows the bicoherence plane of the (a) mag-

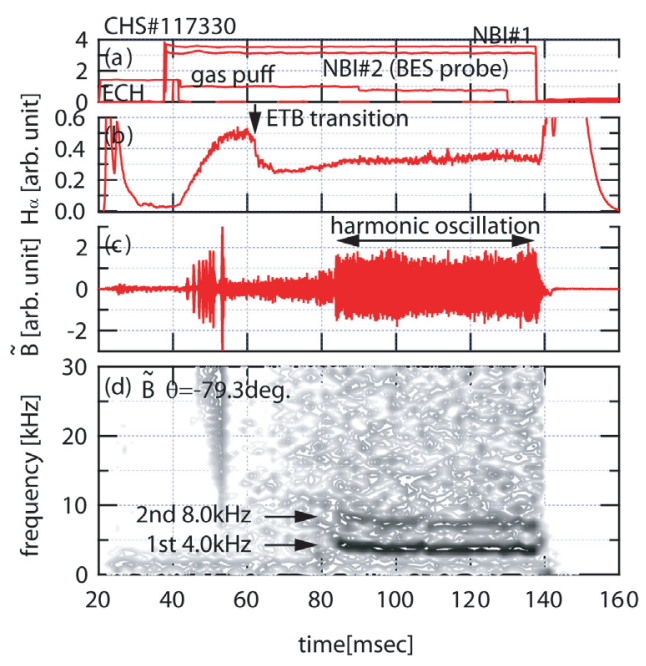

Fig. 1 Typical experimental conditions and temporal evolution of plasma parameters for a discharge in which $\mathrm{HO}$ appears: (a) heating condition, (b) $\mathrm{H}_{\alpha}$ intensity, (c) magnetic fluctuation, and (d) frequency spectrum of magnetic fluctuation. 

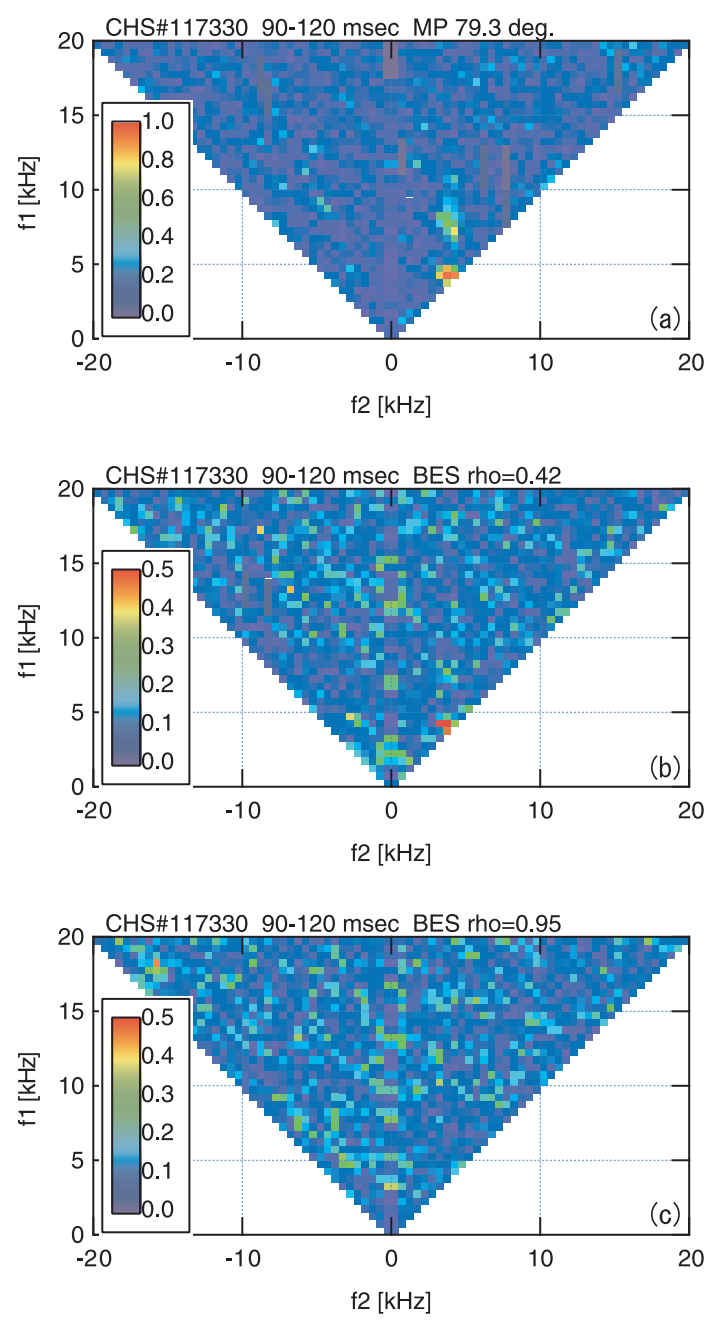

Fig. 2 Bicoherence plane of the (a) magnetic, (b) core $(\rho=$ $0.42)$ density, and (c) edge $(\rho=0.95)$ density fluctuations. $b^{2}\left(f_{1}, f_{2}\right)$ of both the magnetic and core density fluctuations have a clear peak with frequencies $\left(f_{1}, f_{2}\right)=(4.0 \mathrm{kHz}, 4.0 \mathrm{kHz})$, while that of the edge density fluctuation does not have such a peak.

netic, (b) core $(\rho=0.42)$ density, and (c) edge ( $\rho=0.95)$ density fluctuations. $b^{2}\left(f_{1}, f_{2}\right) s$ of both the magnetic and core density fluctuations have a clear peak with the frequencies $\left(f_{1}, f_{2}\right)=(4.0 \mathrm{kHz}, 4.0 \mathrm{kHz})$. This indicates that the 1 st component of $\mathrm{HO}$ (core) with the frequency $4.0 \mathrm{kHz}$ is phase correlated with both the 1st component itself and the 2nd component with the frequency $8.0 \mathrm{kHz}=f_{1}+f_{2}$, where $f_{1}=f_{2}=4.0 \mathrm{kHz}$. On the other hand, $b^{2}\left(f_{1}, f_{2}\right)$ edge density fluctuation does not have such a peak, even though HO (edge) observed in BES signal consists of the 1 st frequency of $4.0 \mathrm{kHz}$ and its 2 nd harmonic. This suggests that phase correlation between harmonic components seems to be different between HO (core) and HO (edge).

We have found that $b^{2}\left(f_{1}, f_{2}\right)$, which indicates phase correlation between harmonic components, has a poloidal asymmetry. Figure 3 shows the frequency spectrum of the magnetic fluctuation with up to 3rd harmonic of HO. The 1st, 2nd, and 3rd frequency $f(1 \mathrm{st}), f(2 \mathrm{nd})$, and $f(3 \mathrm{rd})$ is $3.0,6.5$, and $10.0 \mathrm{kHz}$, respectively. The bicoherence

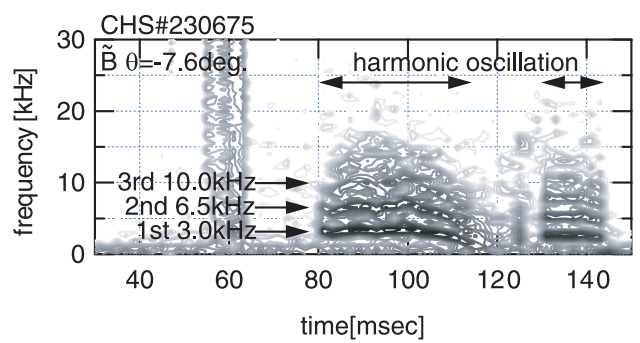

Fig. 3 Frequency spectrum of the magnetic fluctuation with up to 3rd harmonic of $\mathrm{HO}$.
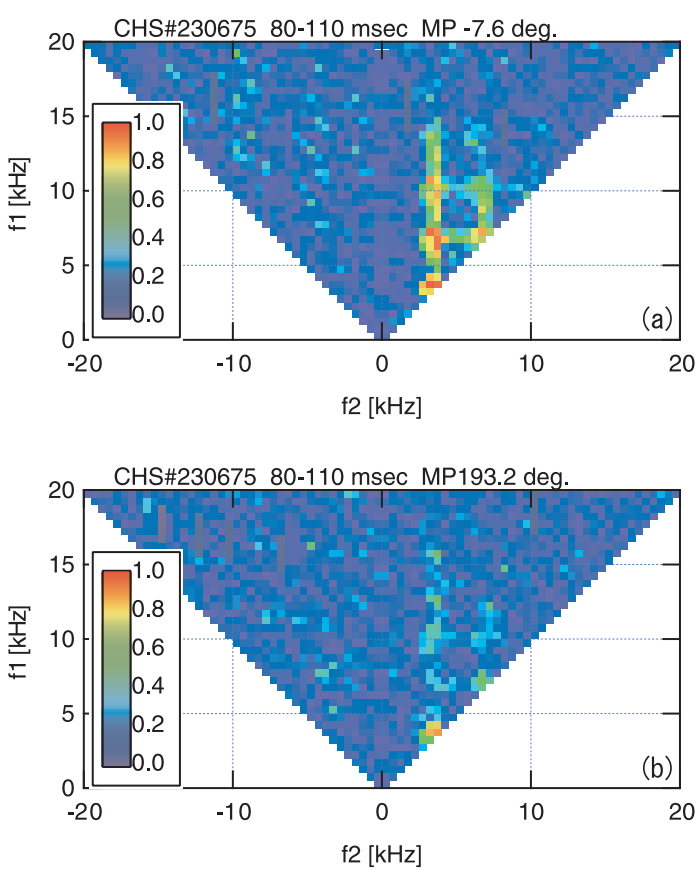

Fig. 4 Bicoherence plane of the magnetic fluctuations (a) outside the torus and (b) inside the torus with frequencies of $\mathrm{HO} f(1 \mathrm{st})=3.0 \mathrm{kHz}, f(2 \mathrm{nd})=6.5 \mathrm{kHz}$, and $f$ $(3 \mathrm{rd})=10.0 \mathrm{kHz} . b^{2}\left(f_{1}, f_{2}\right)$ outside the torus has peaks with $\left(f_{1}, f_{2}\right)=(f(1 \mathrm{st}), f(1 \mathrm{st})),(f(2 \mathrm{nd}), f(1 \mathrm{st})),(f(3 \mathrm{rd})$, $f(1 \mathrm{st})),(f(2 \mathrm{nd}), f(2 \mathrm{nd}))$, and so on, which indicates the phase correlation between each harmonic component. $b^{2}\left(f_{1}, f_{2}\right)$ inside the torus has peaks located at similar frequencies as those outside the torus; however, the values are small.

planes of magnetic fluctuations (a) outside the torus and (b) inside the torus are shown in Fig. $4 . b^{2}\left(f_{1}, f_{2}\right)$ outside the torus has peaks with $\left(f_{1}, f_{2}\right)=(f(1 \mathrm{st}), f(1 \mathrm{st}))$, ( $f(2 \mathrm{nd}), f(1 \mathrm{st})),(f(3 \mathrm{rd}), f(1 \mathrm{st})),(f(2 \mathrm{nd}), f(2 \mathrm{nd}))$, and so on, which indicates the phase correlation between each harmonic component. On the other hand, $b^{2}\left(f_{1}, f_{2}\right)$ inside the torus also has peaks located at frequencies similar to those outside the torus; however, the values are small. Figure 5 shows (a) $b^{2}\left(f_{1}, f_{2}\right)$ at $\left(f_{1}, f_{2}\right)=(f(1 \mathrm{st}), f(1 \mathrm{st}))$ and ( $f(2 \mathrm{nd}), f(1 \mathrm{st}))$ and (b) amplitudes of the $2 \mathrm{nd}(f(2 \mathrm{nd})$ $=f(1 \mathrm{st})+f(1 \mathrm{st}))$ and 3rd $(f(3 \mathrm{rd})=f(2 \mathrm{nd})+f(1 \mathrm{st}))$ harmonic of $\mathrm{HO}$ divided by that of the 1 st harmonic with respect to poloidal angles. One can see that relative amplitudes of higher harmonics decrease with decreasing corresponding $b^{2}\left(f_{1}, f_{2}\right)$ in the poloidal direction. 
We should examine the physical interpretation of the result of the bispectral analysis. In the present study, $b^{2}\left(f_{1}, f_{2}\right)$ has peaks at $f(1 \mathrm{st})+f(1 \mathrm{st})=f(2 \mathrm{nd})$ and $f(2 \mathrm{nd})$ $+f(1 \mathrm{st})=f(3 \mathrm{rd})$. However, the poloidal/toroidal mode number $m / n$ does not satisfy the summation rule, i.e., they show $m(1 \mathrm{st})=m(2 \mathrm{nd})=m(3 \mathrm{rd})=-2$ and $n(1 \mathrm{st})=n(2 \mathrm{nd})$ $=n(3 \mathrm{rd})=1$ [23]. Therefore, generation of harmonics cannot be interpreted simply as mode coupling. $b^{2}\left(f_{1}, f_{2}\right)$ can be regarded as the degree of "distortion" of the signals of $\mathrm{HO}$ (core) in the magnetic and density fluctuations which shows significant phase correlation between harmonic components, as indicated in the poloidal asymmetry of $b^{2}\left(f_{1}, f_{2}\right)$ and relative amplitude of higher harmonics. We suspect that one of the reasons for the signals distortion is the effect of the shape of plasma. In our previous study, we conducted an experiment while scanning the magnitude of the quadrupole field $B_{\mathrm{q}}$ to modify the shape of poloidal cross section. It revealed that the relative amplitude of higher harmonics in the magnetic fluctuation changed monotonically with ellipticity [24]. On the other hand, significant phase correlation was not observed between the harmonic components of HO (edge), even if the signal has harmonic components, i.e., the signal is distorted. In this case, $b^{2}\left(f_{1}, f_{2}\right)$ would not be an appropriate indicator to characterize the signal. As mentioned above, several different characteristics between $\mathrm{HO}$ (core) and (HO) edge have been observed. The mechanism to distort the signal of each oscillation has not been clarified yet, and will be studied in the future.

\section{Summary}

$\mathrm{HO}$ with the fundamental frequency of $3-5 \mathrm{kHz}$ and its higher harmonics has been observed in CHS. It consists of two pairs of harmonic series. One is located in the core region near the $\iota=0.5$ rational surface, the other in the edge region near the $\iota=1.0$ rational surface.

We applied bispectral analysis to the fluctuation data of $\mathrm{HO}$ measured by BES and using MPs. It revealed that fundamental mode of $\mathrm{HO}$ in both the magnetic and core density fluctuations have a phase correlation with the harmonics including fundamental oscillation, while $\mathrm{HO}$ in edge density fluctuation does not have such a phase correlation.

Generation of harmonics cannot be interpreted simply as mode coupling even though bicoherence value $b^{2}\left(f_{1}, f_{2}\right)$ is significant, because mode numbers of HOs for harmonic components with different frequencies are identical. For HO in the core, correlation between $b^{2}\left(f_{1}, f_{2}\right)$ and relative amplitude of higher harmonics suggests that $b^{2}\left(f_{1}, f_{2}\right)$ indicates the degree of distortion of the signals. The reason why the signals are distorted and all harmonic components have identical mode numbers should be investigated in the future.
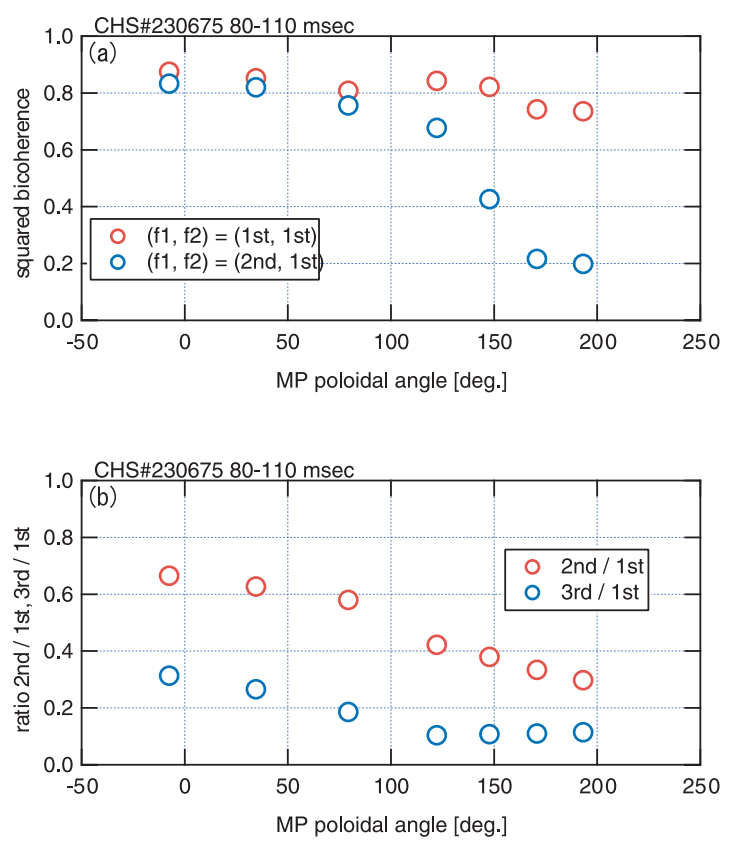

Fig. 5 (a) $b^{2}\left(f_{1}, f_{2}\right)$ at $\left(f_{1}, f_{2}\right)=(f(1 \mathrm{st}), f(1 \mathrm{st}))$ and $(f(2 \mathrm{nd})$, $f(1 \mathrm{st}))$, and (b) amplitudes of the 2nd $(f(2 \mathrm{nd})=$ $f(1 \mathrm{st})+f(1 \mathrm{st}))$ and 3rd $(f(3 \mathrm{rd})=f(2 \mathrm{nd})+f(1 \mathrm{st}))$ harmonic of $\mathrm{HO}$ divided by that of the 1 st harmonic with respect to poloidal angles. Relative amplitudes of harmonics decrease with decrease in the corresponding $b^{2}\left(f_{1}, f_{2}\right)$ in the poloidal direction.

\section{Acknowledgment}

The work was supported in part by the National Institute for Fusion Science Collaborative Research Program (NIFS02KZPD003) directed by the second author.

[1] Y.C. Kim and E.J. Powers, IEEE Trans. Plasma Sci. PS-7, 120 (1979).

[2] Ch. P. Rits et al., Phys. Fluids B 1, 153 (1989).

[3] C. Hidalgo et al., Phys. Rev. Lett. 71, 3127 (1993).

[4] S. Sesnic et al., Nucl. Fusion 34, 1365 (1994).

[5] D. Raju et al., Plasma Phys. Control. Fusion 45, 369 (2003).

[6] Y. Nagashima et al., Phys. Rev. Lett. 95, 095002 (2005).

[7] T. Oishi et al., Nucl. Fusion 46, 317 (2006).

[8] T. Oishi et al., Phys. Plasmas 13, 104504 (2006).

[9] S. Okamura et al., J. Plasma Fusion Res. 79, 977 (2003).

[10] S. Okamura et al., Plasma Phys. Control. Fusion 46, A113 (2004).

[11] T. Minami et al., Plasma Fusion Res. 1, 032 (2006).

[12] T. Akiyama et al., Plasma Phys. Control. Fusion 48, 1683 (2006).

[13] M. Takeuchi et al., Plasma Phys. Control. Fusion 48, A277 (2006).

[14] C.M. Greenfield et al., Phys. Rev. Lett. 86, 4544 (2001).

[15] W. Suttrop et al., Plasma Phys. Control. Fusion 45, 1399 (2003).

[16] W. Suttrop et al., Plasma Phys. Control. Fusion 45, 1399 (2003).

[17] W. Suttrop et al., Nucl. Fusion 45, 721 (2005).

[18] T. Oishi et al., Rev. Sci. Instrum. 75, 4118 (2004).

[19] T. Oishi et al., J. Plasma Fusion Res. SERIES 6, 449 (2004). 
[20] S. Sakakibara et al., Jap. J. Appl. Phys. 63, 4406 (1994)

[21] T. Oishi et al., Plasma Fusion Res. 2, S1097 (2007).

[22] R.J. Fonck et al., Rev. Sci. Instrum. 61, 3487 (1990).
[23] S. Kado et al., J. Nucl. Mat. 363-365, 522 (2007).

[24] S. Kado et al., 21st IAEA Fusion Energy Conference Proceedings, EX/p8-1 (2006). 